\title{
PENYELESAIAN SISTEM PERSAMAAN DIFERENSIAL FRAKSIONAL LINIER ORDE BERBEDA MENGGUNAKAN TRANSFORMASI LAPLACE
}

\author{
NUR AZIZAH, BUDI RUDIANTO* \\ Program Studi S1 Matematika, \\ Fakultas Matematika dan Ilmu Pengetahuan Alam, Universitas Andalas, \\ Kampus UNAND Limau Manis Padang, Indonesia. \\ email : nurazizah110499@gmail.com, budirudianto@sci.unand.ac.id
}

Diterima 15 September 2020 Direvisi 14 Oktober 2020 Dipublikasikan 21 Oktober 2020

\begin{abstract}
Abstrak. Dalam makalah ini diselesaikan sistem persamaan diferensial fraksional linier orde berbeda menggunakan metode transformasi Laplace. Teorema utama yang menyajikan bentuk umum sistem didiskusikan serta beberapa contoh yang mengilustrasikan teorema utama dipaparkan.

Kata Kunci: Sistem Persamaan Diferensial Fraksional Linier Orde Berbeda, Transformasi Laplace
\end{abstract}

\section{Pendahuluan}

Suatu persamaan diferensial adalah suatu persamaan yang memuat turunanturunan dari satu atau lebih variabel terikat terhadap satu atau lebih variabel bebas. Suatu sistem persamaan diferensial adalah kumpulan dari persamaan diferensial. Berdasarkan kelinierannya, sistem persamaan diferensial terbagi atas sistem persamaan diferensial linier dan sistem persamaan diferensial non linier. Bentuk umum dari sistem persamaan diferensial linier adalah sebagai berikut:

$$
D_{t} \mathbf{x}(t)=A \mathbf{x}(t)+\mathbf{u}(t), \quad \mathbf{x}(0)=\mathbf{x}_{0}
$$

$\operatorname{dimana} D_{t}=\frac{d}{d t}, A \in \mathbb{R}^{n \times n}, \mathbf{x}(t) \in \mathbb{R}^{n} \operatorname{dan} \mathbf{u}(t) \in \mathbb{R}^{n}$.

Persamaan diferensial fraksional merupakan persamaan diferensial dengan orde riil positif. Bentuk umum dari sistem persamaan diferensial fraksional linier adalah sebagai berikut:

$$
D_{t}^{\alpha} \mathbf{x}(t)=A \mathbf{x}(t)+\mathbf{u}(t), \quad \mathbf{x}(0)=\mathbf{x}_{0}
$$

${ }^{*}$ penulis korespondensi 
dimana $D_{t}^{\alpha}=\frac{d^{\alpha}}{d t^{\alpha}}$ menyatakan operator turunan fraksional. Ada beberapa definisi dari operator turunan fraksional $D_{t}^{\alpha}$. Salah satu yang cukup dikenal adalah operator turunan fraksional Caputo, yang didefinisikan sebagai:

$$
D_{t}^{\alpha} \mathbf{x}(t)=\frac{1}{\Gamma(k-\alpha)} \int_{0}^{t} \frac{D_{\tau}^{(k)} \mathbf{x}(\tau)}{(t-\tau)^{\alpha+1-k}} d \tau
$$

dimana $k-1<\alpha<k, k \in \mathbb{N}$. Solusi dari sistem (1.2) dengan $D_{t}^{\alpha}$ adalah operator turunan Caputo telah diberikan dalam beberapa literatur.

Dalam artikel ini, diselesaikan sistem persamaan diferensial fraksional (1.2) dalam artian $D_{t}^{\alpha} \mathbf{x}(t)=\left[\begin{array}{lll}D_{t}^{\alpha_{1}} x_{1}(t) & D_{t}^{\alpha_{2}} x_{2}(t) \cdots D_{t}^{\alpha_{n}} x_{n}(t)\end{array}\right]^{T}$ dimana $D_{t}^{\alpha_{i}}, i=$ $1,2, \cdots, n, \alpha_{i}$ tak perlu sama dengan $\alpha_{j}$ untuk $j=1,2, \cdots, n$ adalah operator turunan fraksional Caputo dengan menggunakan transformasi Laplace. Penelitian ini mengelaborasi dari yang telah diteliti oleh Odibat, M.Z [6].

Dalam artikel ini akan diselesaikan sistem persamaan diferensial fraksional orde berbeda yang berbentuk

$$
D_{t}^{\alpha} \mathbf{x}(t)=A \mathbf{x}(t), \quad \mathbf{x}(0)=\mathbf{x}_{0}
$$

dengan $D_{t}^{\alpha} \mathbf{x}(t)=\left[\begin{array}{llll}D_{t}^{\alpha_{1}} x_{1}(t) & D_{t}^{\alpha_{2}} x_{2}(t) & \cdots & D_{t}^{\alpha_{n}} x_{n}(t)\end{array}\right]^{T}, 0<\alpha_{i} \leq 1$, dimana $D_{t}^{\alpha_{i}}, i=1,2, \cdots, n$ adalah operator turunan fraksional Caputo dengan menggunakan transformasi Laplace.

\section{Landasan Teori}

Beberapa fungsi yang berperan penting dalam pembahasan sistem persamaan diferensial fraksional linier adalah fungsi Gamma, fungsi Beta dan fungsi MittagLeffler.

Definisi 2.1. [5] Fungsi Gamma dinyatakan sebagai $\Gamma(n)$, didefinisikan sebagai

$$
\Gamma(n)=\int_{0}^{\infty} x^{n-1} e^{-x} d x, \quad n>0 .
$$

Definisi 2.2. [5] Fungsi Beta didefinisikan sebagai

$$
B(p, q)=\int_{0}^{1} x^{p-1}(1-x)^{q-1} d x, x \in \mathbb{R} \text { dan } p, q \in \mathbb{R},
$$

dimana $p>0$ dan $q>0$.

Definisi 2.3. [2] Fungsi Mittag-Leffler satu parameter didefinisikan sebagai

$$
E_{\lambda}(z)=\sum_{k=0}^{\infty} \frac{z^{k}}{\Gamma(\lambda k+1)}
$$

dimana $\lambda>0$ dan $z \in \mathbb{C}$.

Definisi 2.4. [2] Fungsi Mittag-Leffler dua parameter didefinisikan sebagai 


$$
E_{\lambda, \rho}(z)=\sum_{k=0}^{\infty} \frac{z^{k}}{\Gamma(\lambda k+\rho)},
$$

dengan $\lambda>0, \rho>0$ dan $z \in \mathbb{C}$.

Untuk turunan dari fungsi Mittag-Leffler, berlaku

$$
D_{z}^{(m)} E_{\lambda, \rho}(z)=\sum_{k=0}^{\infty} \frac{(k+m) ! z^{k}}{k ! \Gamma(\lambda(k+m)+\rho)}
$$

Dalam artikel ini metode yang digunakan adalah kaidah Cramer. Implementasi metode ini diberikan berikut ini

$$
A \mathbf{x}=\mathbf{b}
$$

dimana $A_{n \times n}$ adalah matriks non singular dengan

$$
\mathbf{x}=\left[\begin{array}{c}
x_{1} \\
x_{2} \\
\vdots \\
x_{n}
\end{array}\right] \text { dan } \mathbf{b}=\left[\begin{array}{c}
b_{1} \\
b_{2} \\
\vdots \\
b_{n}
\end{array}\right]
$$

Jika det $A \neq 0$, solusi dari (2.2) diberikan sebagai berikut

$$
x_{i}=\frac{\operatorname{det}\left(A_{i}\right)}{\operatorname{det}(A)}, i=1,2, \cdots, n,
$$

dimana $A_{i}$ adalah matriks yang diperoleh dengan mengganti kolom ke- $i$ matriks $A$ dengan vektor $\mathbf{b}$.

Turunan biasa yang biasa kita kenal memiliki orde turunan dalam bentuk bilangan bulat positif. Dalam artikel ini akan diperkenalkan turunan fraksional yang ordenya dalam bentuk bilangan riil positif berupa pecahan. Salah satu turunan fraksional yang digunakan yaitu turunan fraksional yang dikembangkan oleh Caputo menggunakan transformasi Laplace.

Definisi 2.5. [5] Misalkan $f(t)$ adalah fungsi sedemikian sehingga $D_{t}^{(k)} f(t)$ ada. Turunan fraksional Caputo orde $\alpha$ dari fungsi $f(t)$ didefinisikan sebagai

$$
D_{t}^{\alpha} f(t)=\frac{1}{\Gamma(k-\alpha)} \int_{0}^{t} \frac{D_{\tau}^{(k)} f(\tau) d \tau}{(t-\tau)^{\alpha+1-k}}
$$

dengan $k-1<\alpha<k, k \in \mathbb{N}$.

Definisi 2.6. [4] Misalkan $f(t)$ adalah suatu fungsi dari $t$. Transformasi Laplace dari $f(t)$, dinyatakan dengan $F(s)$ atau $\mathcal{L}[f(t)]$, didefinisikan sebagai

$$
\mathcal{L}[f(t)]=F(s)=\int_{0}^{\infty} f(t) e^{-s t} d t, s \in \mathbb{C},
$$

asalkan integral tersebut ada.

Definisi 2.7. [8] Konvolusi dari dua buah fungsi yang kontinu $f(t)$ dan $g(t)$ didefinisikan sebagai berikut 
Penyelesaian Sistem Persamaan Diferensial Fraksional Linier Orde Berbeda 321

$$
f(t) * g(t)=\int_{0}^{t} f(t-\tau) g(\tau) d \tau
$$

dimana simbol * menyatakan operator konvolusi.

Teorema 2.8. [5] Jika transformasi Laplace dari fungsi $f(t)$ dan $g(t)$ adalah $F(s)$ dan $G(s)$ maka

$$
\mathcal{L}\left[\int_{0}^{t} f(t-\tau) g(\tau) d \tau\right]=F(s) G(s) .
$$

Bukti.

$$
\begin{aligned}
\mathcal{L}\left[\int_{0}^{t} f(t-\tau) g(\tau) d \tau\right] & =\int_{0}^{\infty}\left[\int_{0}^{t} f(t-\tau) g(\tau) d \tau\right] e^{-s t} d t \\
& =\int_{0}^{\infty} \int_{0}^{\infty} f(t-\tau) g(\tau) e^{-s t} d \tau d t \\
& =\int_{0}^{\infty} \int_{0}^{\infty} f(t-\tau) g(\tau) e^{-s(t-\tau+\tau)} d \tau d t \\
& =\int_{0}^{\infty} \int_{0}^{\infty} f(t-\tau) g(\tau) e^{-s(t-\tau)} e^{-s \tau} d \tau d t \\
& =\int_{0}^{\infty} \int_{0}^{\infty} f(t-\tau) e^{-s(t-\tau)} d(t-\tau) g(\tau) e^{-s \tau} d \tau \\
& =\int_{0}^{\infty} f(t-\tau) e^{-s(t-\tau)} d(t-\tau) \int_{0}^{\infty} g(\tau) e^{-s \tau} d \tau
\end{aligned}
$$

Misalkan $a=t-\tau$, maka

$$
\begin{aligned}
\mathcal{L}\left[\int_{0}^{t} f(t-\tau) g(\tau) d \tau\right] & =\int_{0}^{\infty} f(a) e^{-s a} d a \int_{0}^{\infty} g(\tau) e^{-s \tau} d \tau, \\
& =F(s) G(s) .
\end{aligned}
$$

Teorema 2.9. [8] Misalkan $\mathcal{L}[f(t)]=F(s)$ dan $D_{t}^{\alpha}$ adalah operator turunan fraksional Caputo, maka

$$
\mathcal{L}\left[D_{t}^{\alpha} f(t)\right]=s^{\alpha} F(s)-\sum_{k=1}^{m} s^{(\alpha-k)} D_{t}^{(k-1)} f(0)
$$




\section{Bukti.}

$$
\begin{aligned}
\mathcal{L}\left[D_{t}^{\alpha} f(t)\right] & =\mathcal{L}\left[\frac{1}{\Gamma(m-\alpha)} \int_{0}^{t} \frac{D_{\tau}^{(m)} f(\tau) d \tau}{(t-\tau)^{\alpha+1-m}}\right], \\
& =\frac{1}{\Gamma(m-\alpha)} \mathcal{L}\left[\int_{0}^{t} \frac{D_{\tau}^{(m)} f(\tau) d \tau}{(t-\tau)^{\alpha+1-m}}\right], \\
& =\frac{1}{\Gamma(m-\alpha)} \mathcal{L}\left[\int_{0}^{t}(t-\tau)^{m-1-\alpha} D_{\tau}^{(m)} f(\tau) d \tau\right] .
\end{aligned}
$$

Dengan menggunakan Teorema 2.8 diperoleh

$$
\begin{aligned}
\mathcal{L}\left[D_{t}^{\alpha} f(t)\right] & =\frac{1}{\Gamma(m-\alpha)} \mathcal{L}\left[t^{m-\alpha-1}\right] \mathcal{L}\left[D_{t}^{(m)} f(t)\right] \\
& =\frac{1}{\Gamma(m-\alpha)} \frac{\Gamma(m-\alpha)}{s^{m-\alpha}}\left[s^{m} F(s)-\sum_{k=1}^{m} s^{(m-k)} D_{t}^{(k-1)} f(0)\right] \\
& =\frac{1}{s^{m-\alpha}}\left[s^{m} F(s)-\sum_{k=1}^{m} s^{(m-k)} D_{t}^{(k-1)} f(0)\right] \\
& =s^{\alpha} F(s)-\sum_{k=1}^{m} s^{(\alpha-k)} D_{t}^{(k-1)} f(0) .
\end{aligned}
$$

Teorema 2.10. [9] Transformasi Laplace dari perkalian fungsi $t^{(\lambda m+\rho-1)} D_{t}^{(m)} E_{\lambda, \rho}\left(a t^{\lambda}\right)$ adalah

$$
\mathcal{L}\left[t^{(\lambda m+\rho-1)} D_{t}^{(m)} E_{\lambda, \rho}\left(a t^{\lambda}\right)\right]=\frac{m ! s^{\lambda-\rho}}{\left(s^{\lambda}-a\right)^{m+1}}, \quad s^{\lambda} \neq a
$$

Bukti. Bukti teorema ini menggunakan formula

$$
\sum_{k=0}^{\infty} \frac{(k+m) !}{k !} x^{k}=\frac{m !}{(1-x)^{m+1}}
$$

yang buktinya akan diberikan kemudian. Dari formula (2.1) diperoleh

$$
D_{t}^{(m)} E_{\lambda, \rho}\left(a t^{\lambda}\right)=\sum_{k=0}^{\infty} \frac{(k+m) !}{k !} \frac{\left(a t^{\lambda}\right)^{k}}{\Gamma(\lambda k+\lambda m+\rho)}
$$


Selanjutnya

$$
\begin{aligned}
\mathcal{L}\left[t^{(\lambda m+\rho-1)} D_{t}^{(m)} E_{\lambda, \rho}\left(a t^{\lambda}\right)\right] & =\int_{0}^{\infty} e^{-s t} t^{(\lambda m+\rho-1)} D_{t}^{(m)} E_{\lambda, \rho}\left(a t^{\lambda}\right) d t \\
& =\int_{0}^{\infty} e^{-s t} t^{(\lambda m+\rho-1)} \sum_{k=0}^{\infty} \frac{(k+m) !}{k !} \frac{\left(a t^{\lambda}\right)^{k}}{\Gamma(\lambda k+\lambda m+\rho)} d t \\
& =\sum_{k=0}^{\infty} \frac{(k+m) !}{k !} \frac{a^{k}}{\Gamma(\lambda k+\lambda m+\rho)} \int_{0}^{\infty} e^{-s t} t^{(\lambda k+\lambda m+\rho-1)} d t \\
& =\sum_{k=0}^{\infty} \frac{(k+m) !}{k !} \frac{a^{k}}{\Gamma(\lambda k+\lambda m+\rho)} \mathcal{L}\left[t^{(\lambda k+\lambda m+\rho-1)}\right] \\
& =\sum_{k=0}^{\infty} \frac{(k+m) !}{k !} \frac{a^{k}}{\Gamma(\lambda k+\lambda m+\rho)} \frac{\Gamma(\lambda k+\lambda m+\rho)}{s^{\lambda k+\lambda m+\rho}}, \\
& =\sum_{k=0}^{\infty} \frac{(k+m) ! a^{k}}{k ! s^{\lambda k+\lambda m+\rho},} \\
& =s^{-(\lambda m+\rho)} \sum_{k=0}^{\infty} \frac{(k+m) !\left(a s^{-\lambda}\right)^{k}}{k !}
\end{aligned}
$$

Dengan menggunakan persamaan (2.4), diperoleh

$$
\begin{aligned}
\mathcal{L}\left[t^{(\lambda m+\rho-1)} D_{t}^{(m)} E_{\lambda, \rho}\left(a t^{\lambda}\right)\right] & =s^{-(\lambda m+\rho)} \frac{m !}{\left(1-a s^{-\lambda}\right)^{m+1}}, \\
& =s^{-(\lambda m+\rho)} s^{\lambda(m+1)} \frac{m !}{\left(s^{\lambda}-a\right)^{m+1}}, \\
& =\frac{m ! s^{\lambda-\rho}}{\left(s^{\lambda}-a\right)^{m+1}} .
\end{aligned}
$$

Selanjutnya akan dibuktikan bahwa

$$
\sum_{k=0}^{\infty} \frac{(k+m) !}{k !} x^{k}=\frac{m !}{(1-x)^{m+1}} .
$$

Terlebih dahulu akan dibuktikan bahwa

$$
(1+x)^{m}=\sum_{k=0}^{\infty}\left(\begin{array}{c}
m \\
k
\end{array}\right) x^{k},
$$

dan

$$
\left(\begin{array}{c}
-n \\
r
\end{array}\right)=(-1)^{r} C_{(n+r-1, r)} .
$$

Misalkan $f(x)=(1+x)^{m}$, maka

$$
\begin{aligned}
f(x) & =(1+x)^{m} \rightarrow f(0)=1, \\
D_{x} f(x) & =m(1+x)^{m-1} \rightarrow D_{x} f(0)=m, \\
D_{x}^{2} f(x) & =m(m-1)(1+x)^{m-2} \rightarrow D_{x}^{2} f(0)=m(m-1), \\
D_{x}^{3} f(x) & =m(m-1)(m-2)(1+x)^{m-3} \rightarrow D_{x}^{3} f(0)=m(m-1)(m-2) .
\end{aligned}
$$


324 Nur Azizah

Dengan menggunakan Deret Maclaurin, yaitu

$$
f(x)=f(0)+\frac{x}{1 !} D_{x} f(0)+\frac{x}{2 !} D_{x}^{2} f(0)+\frac{x}{3 !} D_{x}^{3} f(0)+\cdots,
$$

diperoleh

$$
\begin{aligned}
f(x) & =1+\frac{x}{1 !} m+\frac{x^{2}}{2 !} m(m-1)+\frac{x^{3}}{3 !} m(m-1)(m-2)+\cdots, \\
& =x^{0}+m x+\frac{x^{2}}{2 !} m(m-1) \frac{(m-2) !}{(m-2) !}+\frac{x^{3}}{3 !} m(m-1)(m-2) \frac{(m-3) !}{(m-3) !}+\cdots, \\
& =x^{0}+m x+\frac{m !}{2 !(m-2) !} x^{2}+\frac{m !}{3 !(m-3) !} x^{3}+\cdots, \\
& =\left(\begin{array}{c}
m \\
0
\end{array}\right) x^{0}+\left(\begin{array}{c}
m \\
1
\end{array}\right) x+\left(\begin{array}{c}
m \\
2
\end{array}\right) x^{2}+\left(\begin{array}{c}
m \\
3
\end{array}\right) x^{3}+\cdots, \\
f(x) & =\sum_{k=0}^{\infty}\left(\begin{array}{c}
m \\
k
\end{array}\right) x^{k} .
\end{aligned}
$$

Kemudian,

$$
\begin{aligned}
\left(\begin{array}{c}
-n \\
r
\end{array}\right) & =\frac{(-n) !}{r !(n-r) !}, \\
& =\frac{(-n)(-n-1)(-n-2) \cdots(-n-(r-1))(-n-r) !}{r !(-n-r) !}, \\
& =\frac{(-n)(-n-1)(-n-2) \cdots(-n-r+1)}{r !}, \\
& =\frac{(-1)^{r} n(n+1)(n+2) \cdots(n+r-1)}{r !}, \\
& =\frac{(-1)^{r}(n+r-1)(n+r-2) \cdots(n+1) n}{r !}, \\
& =\frac{(-1)^{r}(n+r-1)(n+r-2) \cdots(n+1) n}{r !} \frac{(n-1) !}{(n-1) !} \\
& =\frac{(-1)^{r}(n+r-1) !}{r !(n-1) !}, \\
& =(-1)^{r}\left(\begin{array}{c}
n+r-1 \\
r
\end{array}\right) \\
\left(\begin{array}{c}
-n \\
r
\end{array}\right) & =(-1)^{r} C_{(n+r-1, r) .}
\end{aligned}
$$


Dari persamaan (2.6) dan (2.7) diperoleh

$$
\begin{aligned}
(1+x)^{-m} & =\sum_{k=0}^{\infty}(-1)^{k}\left(\begin{array}{c}
m+k-1 \\
k
\end{array}\right) x^{k}, \\
(1-x)^{-m} & =\sum_{k=0}^{\infty}(-1)^{k}\left(\begin{array}{c}
m+k-1 \\
k
\end{array}\right)(-x)^{k}, \\
& =\sum_{k=0}^{\infty}\left(\begin{array}{c}
m+k-1 \\
k
\end{array}\right) x^{k}, \\
(1-x)^{-(m+1)} & =\sum_{k=0}^{\infty}\left(\begin{array}{c}
m+1)+k-1 \\
k
\end{array}\right) x^{k}, \\
& =\sum_{k=0}^{\infty}\left(\begin{array}{c}
m+k \\
k
\end{array}\right) x^{k} .
\end{aligned}
$$

Dengan demikian diperoleh

$$
\begin{aligned}
\frac{1}{(1-x)^{(m+1)}} & =\sum_{k=0}^{\infty}\left(\begin{array}{c}
m+k \\
k
\end{array}\right) x^{k}, \\
& =\sum_{k=0}^{\infty} \frac{(m+k) !}{(m+k-k) ! k !} x^{k}, \\
& =\sum_{k=0}^{\infty} \frac{(m+k) !}{m ! k !} x^{k}, \\
& =\frac{1}{m !} \sum_{k=0}^{\infty} \frac{(m+k) !}{k !} x^{k}, \\
\frac{m !}{(1-x)^{(m+1)}} & =\sum_{k=0}^{\infty} \frac{(m+k) !}{k !} x^{k} .
\end{aligned}
$$

\section{Pembahasan}

Perhatikan kembali sistem persamaan (1.4). Misalkan

$$
A=\left[\begin{array}{cccc}
a_{11} & a_{12} & \cdots & a_{1 n} \\
a_{21} & a_{22} & \cdots & a_{2 n} \\
\vdots & & & \\
a_{n 1} & a_{n 2} & \cdots & a_{n n}
\end{array}\right] \text { dan } \mathbf{x}=\left[\begin{array}{c}
x_{1} \\
x_{2} \\
\vdots \\
x_{n}
\end{array}\right]
$$

Persamaan (1.4) dapat ditulis menjadi

$$
\left\{\begin{aligned}
D_{t}^{\alpha_{1}} x_{1}(t) & =a_{11} x_{1}(t)+a_{12} x_{2}(t)+\cdots+a_{1 n} x_{n}(t) \\
D_{t}^{\alpha_{2}} x_{2}(t) & =a_{21} x_{1}(t)+a_{22} x_{2}(t)+\cdots+a_{2 n} x_{n}(t) \\
& \vdots \\
D_{t}^{\alpha_{n}} x_{n}(t) & =a_{n 1} x_{1}(t)+a_{n 2} x_{2}(t)+\cdots+a_{n n} x_{n}(t)
\end{aligned}\right.
$$


326 Nur Azizah

dengan syarat awal :

$$
x_{1}(0)=x_{10}, x_{2}(0)=x_{20}, \cdots, x_{n}(0)=x_{n 0} .
$$

Dengan melakukan transformasi Laplace pada (3.2), diperoleh

$$
\left\{\begin{aligned}
s^{\alpha_{1}} X_{1}(s)-s^{\alpha_{1}-1} x_{1}(0) & =a_{11} X_{1}(s)+a_{12} X_{2}(s)+\cdots+a_{1 n} X_{n}(s) \\
s^{\alpha_{2}} X_{2}(s)-s^{\alpha_{2}-1} x_{2}(0) & =a_{21} X_{1}(s)+a_{22} X_{2}(s)+\cdots+a_{2 n} X_{n}(s) \\
& \vdots \\
s^{\alpha_{n}} X_{n}(s)-s^{\alpha_{n}-1} x_{n}(0) & =a_{n 1} X_{1}(s)+a_{n 2} X_{2}(s)+\cdots+a_{n n} X_{n}(s)
\end{aligned}\right.
$$

Dengan demikian diperoleh

$$
\left\{\begin{array}{c}
\left(a_{11}-s^{\alpha_{1}}\right) X_{1}(s)+a_{12} X_{2}(s)+\cdots+a_{1 n} X_{n}(s)=-s^{\alpha_{1}-1} x_{1}(0) \\
a_{21} X_{1}(s)+\left(a_{22}-s^{\alpha_{2}}\right) X_{2}(s)+\cdots+a_{2 n} X_{n}(s)=-s^{\alpha_{2}-1} x_{2}(0) \\
\vdots \\
a_{n 1} X_{1}(s)+a_{n 2} X_{2}(s)+\cdots+\left(a_{n n}-s^{\alpha_{n}}\right) X_{n}(s)=-s^{\alpha_{n}-1} x_{n}(0) .
\end{array}\right.
$$

Persamaan (3.4) dapat ditulis menjadi

$$
B(s) \mathbf{X}(s)=\mathbf{L}(s),
$$

dimana

$$
B(s)=\left[\begin{array}{cccc}
a_{11}-s^{\alpha_{1}} & a_{12} & \cdots & a_{1 n} \\
a_{21} & a_{22}-s^{\alpha_{2}} & \cdots & a_{2 n} \\
\vdots & & & \\
a_{n 1} & a_{n 2} & \cdots & a_{n n}-s^{\alpha_{n}}
\end{array}\right], \mathbf{X}(s)=\left[\begin{array}{c}
X_{1}(s) \\
X_{2}(s) \\
\vdots \\
X_{n}(s)
\end{array}\right]
$$

dan

$$
\mathbf{L}(s)=\left[\begin{array}{c}
-s^{\alpha_{1}-1} x_{1}(0) \\
-s^{\alpha_{2}-1} x_{2}(0) \\
\vdots \\
-s^{\alpha_{n}-1} x_{n}(0)
\end{array}\right]
$$

Misalkan $B(s)$ adalah matriks non singular, maka dengan menggunakan aturan Cramer, diperoleh

$$
X_{j}(s)=\frac{\operatorname{det}\left(B_{j}(s)\right)}{\operatorname{det}(B(s))}, \text { untuk } j=1,2, \cdots, n
$$

dimana $B_{j}(s)$ adalah matriks yang dibentuk dengan mengganti kolom ke-j dari matriks $B(s)$ dengan vektor $\mathbf{L}(s)$. Dari persamaan (3.6) diperoleh

$$
x_{j}(t)=\mathcal{L}^{-1}\left[\frac{\operatorname{det}\left(B_{j}(s)\right)}{\operatorname{det}(B(s))}\right], j=1,2, \cdots, n .
$$

Contoh 3.1. Tentukan solusi dari sistem

$$
\left[\begin{array}{c}
D_{t}^{1 / 2} x(t) \\
D_{t}^{1 / 3} y(t)
\end{array}\right]=\left[\begin{array}{cc}
1 / 8 & 0 \\
a & -1
\end{array}\right]\left[\begin{array}{l}
x \\
y
\end{array}\right],\left[\begin{array}{l}
x(0) \\
y(0)
\end{array}\right]=\left[\begin{array}{l}
x_{0} \\
y_{0}
\end{array}\right]
$$

dimana $a \in \mathbb{R}$. 
Dengan menerapkan transformasi Laplace terhadap persamaan (3.8), diperoleh:

$$
\begin{aligned}
& -s^{-1 / 2} x(0)=\left(\frac{1}{8}-s^{1 / 2}\right) X(s), \\
& -s^{-2 / 3} y(0)=a X(s)-\left(1+s^{1 / 3}\right) Y(s) .
\end{aligned}
$$

Persamaan (3.9) dan (3.10) dapat ditulis menjadi

$$
\left[\begin{array}{l}
-s^{-1 / 2} x(0) \\
-s^{-2 / 3} y(0)
\end{array}\right]=\left[\begin{array}{cc}
\frac{1}{8}-s^{1 / 2} & 0 \\
a & -1-s^{1 / 3}
\end{array}\right]\left[\begin{array}{l}
X(s) \\
Y(s)
\end{array}\right] .
$$

Maka

$$
X(s)=\frac{s^{-1 / 2}}{s^{1 / 2}-\frac{1}{8}} x_{0}
$$

Dengan menggunakan Teorema 2.10, diperoleh

$$
x(t)=E_{1 / 2,1}\left(\frac{1}{8} t^{1 / 2}\right) x_{0} .
$$

Selanjutnya

$$
Y(s)=a \frac{s^{-1 / 2}}{\left(s^{1 / 2}-\frac{1}{8}\right)\left(s^{1 / 3}+1\right)} x_{0}+\frac{s^{-2 / 3}}{\left(s^{1 / 3}+1\right)} y_{0} .
$$

Dengan menggunakan Teorema 2.10, diperoleh

$$
\begin{aligned}
y(t)= & a t^{-1 / 3}\left[\frac{16}{15} E_{1 / 6,2 / 3}\left(\frac{1}{2} t^{1 / 6}\right)-\frac{40-24 \sqrt{3} i}{39} E_{1 / 6,2 / 3}\left(\frac{-1+\sqrt{3} i}{4} t^{1 / 6}\right)\right. \\
& -\frac{40+24 \sqrt{3} i}{39} E_{1 / 6,2 / 3}\left(\frac{-1-\sqrt{3} i}{4} t^{1 / 6}\right)+\frac{32-4 i}{65} E_{1 / 6,2 / 3}\left(-i t^{1 / 6}\right) \\
& \left.+\frac{32+4 i}{65} E_{1 / 6,2 / 3}\left(i t^{1 / 6}\right)\right] x_{0}+E_{1 / 3}\left(-t^{1 / 3}\right) y_{0} .
\end{aligned}
$$

Contoh 3.2. Tentukan solusi dari sistem

$$
\left[\begin{array}{c}
D_{t} x(t) \\
D_{t}^{1 / 2} y(t) \\
D_{t}^{1 / 2} z(t)
\end{array}\right]=\left[\begin{array}{ccc}
-2 & 0 & 0 \\
0 & 5 & 0 \\
0 & 4 & 5
\end{array}\right]\left[\begin{array}{l}
x \\
y \\
z
\end{array}\right],\left[\begin{array}{l}
x(0) \\
y(0) \\
z(0)
\end{array}\right]=\left[\begin{array}{l}
x_{0} \\
y_{0} \\
z_{0}
\end{array}\right]
$$

Dengan menerapkan transformasi Laplace terhadap persamaan (3.11), diperoleh:

$$
-x(0)=(-2-s) X(s)
$$

dan

$$
-s^{-1 / 2} y(0)=\left(5-s^{1 / 2}\right) Y(s)
$$

dan

$$
-s^{-1 / 2} z(0)=4 Y(s)+\left(5-s^{1 / 2}\right) Z(s) .
$$


Persamaan (3.12), (3.13) dan (3.14) dapat ditulis menjadi:

$$
\left[\begin{array}{c}
-x(0) \\
-s^{-1 / 2} y(0) \\
-s^{-1 / 2} z(0)
\end{array}\right]=\left[\begin{array}{ccc}
-2-s & 0 & 0 \\
0 & 5-s^{1 / 2} & 0 \\
0 & 4 & 5-s^{1 / 2}
\end{array}\right]\left[\begin{array}{l}
X(s) \\
Y(s) \\
Z(s)
\end{array}\right],
$$

maka

$$
X(s)=\frac{1}{s+2} x_{0} .
$$

Dengan menggunakan Teorema 2.10, diperoleh

$$
x(t)=\exp (-2 t) x_{0} .
$$

Selanjutnya

$$
Y(s)=\frac{s^{-1 / 2}}{s^{1 / 2}-5} y_{0} .
$$

Dengan menggunakan Teorema 2.10, diperoleh

$$
y(t)=E_{1 / 2,1}\left(5 t^{1 / 2}\right) y_{0} .
$$

Selanjutnya

$$
Z(s)=\frac{4 s^{-1 / 2}}{\left(s^{1 / 2}-5\right)^{2}} y_{0}+\frac{s^{-1 / 2}}{s^{1 / 2}-5} z_{0} .
$$

Dengan menggunakan Teorema 2.10, diperoleh

$$
z(t)=4 t^{1 / 2} D_{t} E_{1 / 2,1}\left(5 t^{1 / 2}\right) y_{0}+E_{1 / 2,1}\left(5 t^{1 / 2}\right) z_{0} .
$$

\section{Kesimpulan}

Penyelesaian dari sistem persamaan diferensial fraksional orde berbeda

$$
D_{t}^{\alpha} \mathbf{x}(t)=A \mathbf{x}(t), \quad \mathbf{x}(0)=\mathbf{x}_{0},
$$

dengan $D_{t}^{\alpha} \mathbf{x}(t)=\left[\begin{array}{llll}D_{t}^{\alpha_{1}} x_{1}(t) & D_{t}^{\alpha_{2}} x_{2}(t) & \cdots & D_{t}^{\alpha_{n}} x_{n}(t)\end{array}\right]^{T}, 0<\alpha_{i} \leq 1$, dimana $D_{t}^{\alpha_{i}}, i=1,2, \cdots, n$ dengan $D_{t}^{\alpha}$ adalah operator turunan fraksional Caputo adalah

$$
x_{j}(t)=\mathcal{L}^{-1}\left[\frac{\operatorname{det}\left(B_{j}(s)\right)}{\operatorname{det}(B(s))}\right], j=1,2, \cdots, n .
$$

dimana

$$
B(s)=\left[\begin{array}{cccc}
a_{11}-s^{\alpha_{1}} & a_{12} & \cdots & a_{1 n} \\
a_{21} & a_{22}-s^{\alpha_{2}} & \cdots & a_{2 n} \\
\vdots & & & \\
a_{n 1} & a_{n 2} & \cdots & a_{n n}-s^{\alpha_{n}}
\end{array}\right], \mathbf{L}(s)=\left[\begin{array}{c}
-s^{\alpha_{1}-1} x_{1}(0) \\
-s^{\alpha_{2}-1} x_{2}(0) \\
\vdots \\
-s^{\alpha_{n}-1} x_{n}(0)
\end{array}\right]
$$

dan $B_{j}(s)$ adalah matriks yang dibentuk dengan mengganti kolom ke-j dari matriks $B(s)$ dengan vektor $\mathbf{L}(s)$. 


\section{Ucapan Terima kasih}

Penulis mengucapkan terima kasih kepada Bapak Prof. Dr. Muhafzan, Ibu Dr. Susila Bahri, Bapak Dr. Mahdhivan Syafwan, dan Ibu Dr. Arrival Rince Putri yang telah memberikan masukan dan saran dalam penyempurnaan penulisan artikel ini.

\section{Daftar Pustaka}

[1] Bartle. R. G dan Donald. R. Sherbert., 2010. Introduction to Real Analysis, Fourth Edition. John Wiley and Sons, USA.

[2] Fallahgoul. A, Sergio. Focardi, Frank. Fabozzi. 2017. Fractional Calculus and Fractional Processes with Applications to Financial Economics. Academic Press, Australia.

[3] Kaczorek, T. 2011. Selected Problem of Fractional Systems Theory. SpringerVerlag, Berlin Heidelberg.

[4] Kontributor Wikipedia. "Laplace Transform." Wikipedia, Ensiklopedia Bebas. Wikipedia, Ensiklopedia Bebas, 24 Sept. 2020. Web. 24 Sept. 2020.

[5] Milici. Constantin, Gheorghe. D, J. Tanreiro. 2019. Introduction to Fractional Differential Equations. Springer, Switzerland.

[6] Odibat, M. Z. 2009. Analytic Study on Linear Systems of Fractional Differential Equations. Computers and Mathematics with Applications. Elsevier Ltd.

[7] Poole, David. 2005. Linear Algebra A Modern Introduction. Brooks Cole, New York.

[8] Schiff, J. L. 1999. The Laplace Transform. Springer-Verlag, New York.

[9] Spiegel, M. R. 1999. Transformasi Laplace. Terjemahan Bahasa Indonesia. Erlangga, Jakarta. 